\title{
UK Renal Registry 19th Annual Report: Appendix I Acronyms and Abbreviations used in the Annual Report
}

\author{
$\mathrm{AAB}$ \\ ACE (inhibitor) \\ AKI \\ ANZDATA \\ APD \\ ADPKD \\ APKD \\ ATTOM \\ ATTOMic \\ AV \\ AVF \\ AVG \\ BAPN \\ BCG \\ BCP \\ Bicarb \\ BMD \\ BMI \\ BP \\ BSI \\ BTS \\ $\mathrm{Ca}$ \\ $\mathrm{CAB}$ \\ CABG \\ CAPD \\ CCG \\ CCL \\ CCPD \\ CDI \\ Chol \\ $\mathrm{CHr}$ \\ CI \\ CICR \\ CIF \\ CK \\ CKD \\ CKD-EPI \\ CK-MB \\ CKD-MBD \\ COPD \\ Academic Affairs Board (Renal Association) \\ Angiotensin converting enzyme (inhibitor) \\ Acute kidney injury \\ Australia and New Zealand Dialysis and Transplant Registry \\ Automated peritoneal dialysis \\ Autosomal dominant polycystic kidney disease \\ Adult polycystic kidney disease \\ Access to transplant and transplant outcome measures \\ Access to transplant and transplant outcome measures in children \\ Arteriovenous \\ Arteriovenous fistula \\ Arteriovenous graft \\ British Association of Paediatric Nephrology \\ Bromocresol green \\ Bromocresol purple \\ Bicarbonate \\ Bone mineral disease \\ Body mass index \\ Blood pressure \\ Blood stream infection \\ British Transplant Society \\ Calcium \\ Clinical Affairs Board (Renal Association) \\ Coronary artery bypass grafting \\ Continuous ambulatory peritoneal dialysis \\ Clinical Commissioning Group \\ Clinical Computing Limited \\ Cycling peritoneal dialysis \\ Clostridium difficile infection \\ Cholesterol \\ Target reticulocyte $\mathrm{Hb}$ content \\ Confidence interval \\ Cumulative incidence competing risk \\ Cumulative incidence function \\ Creatine kinase \\ Chronic kidney disease \\ Chronic kidney disease epidemiology collaboration \\ Creatine kinase isoenzyme MB \\ Chronic kidney disease- mineral bone disorder \\ Chronic obstructive pulmonary disease
}

\section{KARGER}

Fax +4161306 1234 E-Mail karger@karger.com www.karger.com/nef
C 2017 The UK Renal Registry

Published by S. Karger AG, Basel

Karger
Open access

This article is licensed under the Creative Commons AttributionNonCommercial-NoDerivatives 4.0 International License (CC BYNC-ND) (http://www karger.com/Services/OpenAccessLicense) Us-ND) (http://www.karger.com/Services/OpenAccessLicense). Usage and distribution for commercial purposes as well as any
distribution of modified material requires written permission.
UK Renal Registry, Southmead Hospital, Southmead Road, Bristol, BS10 5NB, UK

Email: renalregistry@renalregistry.nhs.uk 


\begin{tabular}{|c|c|}
\hline Creat & Creatinine \\
\hline $\mathrm{cRF}$ & Calculated HLA antibody reaction frequency \\
\hline CRF & Chronic renal failure \\
\hline CRP & C-reactive protein \\
\hline CRVF & Cardiovascular risk factor \\
\hline CVVH & Continuous veno-venous haemofiltration \\
\hline CXR & Chest $\mathrm{x}$-ray \\
\hline DBP & Diastolic blood pressure \\
\hline DCCT & Diabetes Control and Complications Trial \\
\hline DCD & Donor after circulatory death \\
\hline $\mathrm{DH}$ & Department of Health \\
\hline $\mathrm{DM}$ & Diabetes mellitus \\
\hline DOB & Date of birth \\
\hline DOPPS & Dialysis Outcomes and Practice Patterns Study \\
\hline $\mathrm{Ei}$ & Expected cases in area i \\
\hline E Coli & Escherichia coli \\
\hline E\&W & England and Wales \\
\hline $\mathrm{E}, \mathrm{W} \& \mathrm{NI}$ & England, Wales and Northern Ireland \\
\hline EBPG & European Best Practice Guidelines \\
\hline ECG & Electrocardiogram \\
\hline EDTA & European Dialysis and Transplant Association \\
\hline EF & Error factor \\
\hline eGFR & Estimated glomerular filtration rate \\
\hline ECD & Extended Criteria Donor \\
\hline EDTA & European Dialysis and Transplant Association \\
\hline $\mathrm{eKt} / \mathrm{V}$ & Equilibrated $\mathrm{Kt} / \mathrm{V}$ \\
\hline EPO & Erythropoietin \\
\hline ERA & European Renal Association \\
\hline ERA-EDTA & European Renal Association - European Dialysis and Transplant Association \\
\hline ERF & Established renal failure \\
\hline ESA & Erythropoiesis stimulating agent \\
\hline ESRD & End stage renal disease \\
\hline ESRF & End stage renal failure \\
\hline EWNI & England, Wales and Northern Ireland \\
\hline Ferr & Ferritin \\
\hline FEV1 & Forced expiratory volume in 1 second \\
\hline FVC & Forced vital capacity \\
\hline GFR & Glomerular filtration rate \\
\hline $\mathrm{GH}$ & Growth hormone \\
\hline GN & Glomerulonephritis \\
\hline HA & Health Authority \\
\hline $\mathrm{HB}$ & Health board \\
\hline $\mathrm{Hb}$ & Haemoglobin \\
\hline $\mathrm{HbA1c}$ & Glycated Haemoglobin \\
\hline $\mathrm{HBeAg}$ & Hepatitis B e antigen \\
\hline HCAI-DCS & Healthcare-associated infection data collection system \\
\hline $\mathrm{HD}$ & Haemodialysis \\
\hline HDF & Haemodialysis filtration \\
\hline HDL & High-density lipoprotein \\
\hline HES & Hospital Episodes Statistics \\
\hline $\mathrm{HHD}$ & Home haemodialysis \\
\hline HLA & Human leucocyte antigen \\
\hline $\mathrm{HPA}$ & Health Protection Agency \\
\hline HQIP & Health Quality Improvement Partnership \\
\hline $\mathrm{HR}$ & Hazard ratio \\
\hline HRC & Hypochromic red blood cells \\
\hline $\mathrm{Ht}$ & Height \\
\hline HT & Home therapy \\
\hline HTN & Hypertension \\
\hline ICHD & In centre haemodialysis \\
\hline
\end{tabular}




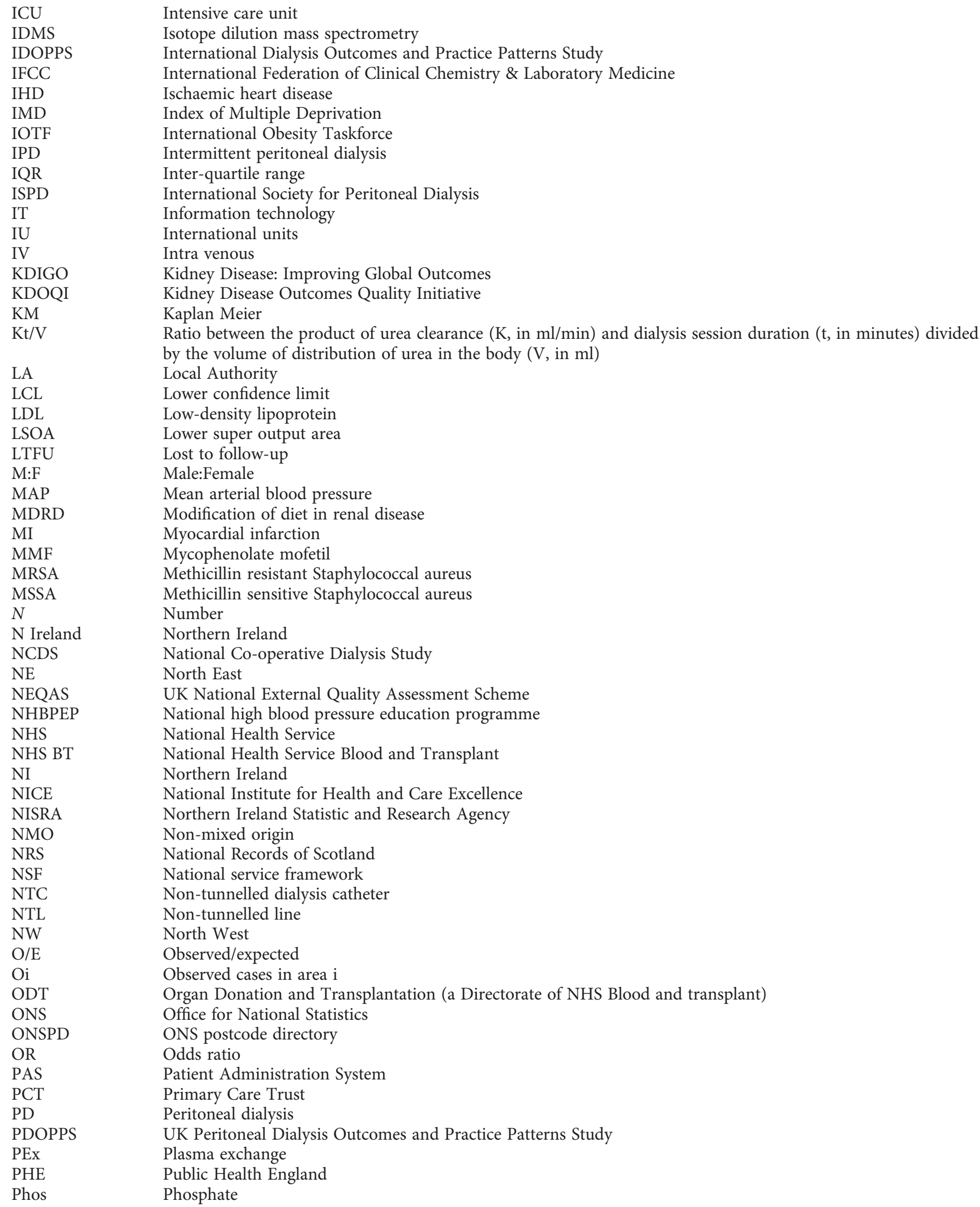




\begin{tabular}{|c|c|}
\hline PIAG & Patient Information Advisory Group \\
\hline PKD & Polycystic kidney disease \\
\hline PMARP & Per million age related population \\
\hline PMCP & Per million child population \\
\hline PMP & Per million population \\
\hline PP & Pulse pressure \\
\hline PRD & Primary renal disease \\
\hline PTH & Parathyroid hormone \\
\hline PTx & Pre-emptive transplant \\
\hline PUV & Posterior urethral valves \\
\hline PVD & Peripheral vascular disease \\
\hline QOF & Quality and Outcomes Framework \\
\hline QUEST & Quality European Studies \\
\hline RA & Renal Association \\
\hline rhGH & Recombinant human growth hormone \\
\hline RI & Royal Infirmary \\
\hline RNSF & Renal National Service Framework (or NSF) \\
\hline $\mathrm{RR}$ & Relative risk \\
\hline RRDSS & RenalRegistry data set specification \\
\hline RRT & Renal replacement therapy \\
\hline RVD & Renovascular disease \\
\hline SAR & Standardised acceptance ratio $(=\mathrm{O} / \mathrm{E})$ \\
\hline SAS & Statistical Analysis System \\
\hline SBP & Systolic blood pressure \\
\hline SD & Standard deviation \\
\hline SES & Socio-economic status \\
\hline SHA & Strategic health authority \\
\hline SHARP & Study of Heart and Renal Protection \\
\hline SI & System International (units) \\
\hline SMR & Standardised mortality ratios \\
\hline $\mathrm{spKt} / \mathrm{V}$ & Single pool Kt/V \\
\hline SPC & Statistical process control \\
\hline SPR & Standardised prevalence ratio $(=\mathrm{O} / \mathrm{E})$ \\
\hline SR & Standardised ratio (used to cover either SAR or SPR) \\
\hline SRR & Scottish Renal Registry \\
\hline SUS & Secondary uses service \\
\hline SW & South West \\
\hline TC & Tunnelled dialysis catheter \\
\hline $\mathrm{TL}$ & Tunnelled line \\
\hline TSAT & Transferrin saturation \\
\hline TWL & Transplant waiting list \\
\hline $\mathrm{Tx}$ & Transplant \\
\hline UCL & Upper confidence limit \\
\hline UK & United Kingdom \\
\hline UKRR & UK Renal Registry \\
\hline UKT & UK Transplant (now ODT) \\
\hline URR & Urea reduction ratio \\
\hline US & United States \\
\hline USA & United States of America \\
\hline USRDS & United States Renal Data System \\
\hline WHO & World health organization \\
\hline $\mathrm{Wt}$ & Weight \\
\hline
\end{tabular}

